\title{
Análisis psicométrico de la Physical Education Season Survey en estudiantes españoles de educación secundaria
}

\section{Rafael BURGUEÑ ${ }^{1}$, María-Jesús LIROLA ${ }^{1}$, Esther MORALES-ORTIZ ${ }^{2}$, Belén CUETO-MARTÍN ${ }^{2}$, Jesús MEDINA-CASAUBÓN ${ }^{2}$}

\author{
Departamento de Educación, Universidad de Almería, España ${ }^{1}$ \\ Departamento de Educación Física y Deportiva, Universidad de Granada, España ${ }^{2}$
}

(Recibido, 14 de octubre, 2017; Aceptado, 27 de noviembre, 2017)

RESUMEN: Tomando como referencia el modelo pedagógico de Educación Deportiva (ED), esta investigación tuvo como objetivo analizar las propiedades psicométricas de la primera sección de la Physical Education Season Survey al objeto de medir las seis características estructurales de la ED en el contexto español. Los participantes fueron 608 estudiantes de educación secundaria obligatoria y post-obligatoria (323 chicos y 285 chicas; $M_{\text {edad }}=14.99, D T=1.58$ ) quienes completaron una intervención de 12 sesiones en clase de Educación Física (EF) de acuerdo con las directrices propuestas para este modelo basado en la práctica. El análisis factorial confirmatorio mostró un buen ajuste para la estructura unidimensional de 6 ítems, la cual permaneció invariante respecto al género y edad. El análisis de consistencia interna reflejó adecuados valores. Los tres análisis de regresión lineal revelaron que la ED predijo de manera positiva la competencia percibida, el compromiso deportivo y el juego limpio, respectivamente. La Physical Education Season Survey se muestra como una medida válida y fiable para evaluar las seis características nucleares de la ED en el contexto español de la EF.

Palabras clave: modelos basados en la práctica, modelos de instrucción deportiva, pedagogía del deporte, evaluación psicométrica, diseño y validación.

\section{Psychometric analysis of the Physical Education Season Survey in Spanish secondary education students}

\begin{abstract}
With reference to the Sport Education pedagogical model, this research aimed to examine the psychometric properties of the first section of the Physical Education Season Survey in order to measure the six structural features of Sport Education in the Spanish context. The participants were 608 Post-Compulsory and Compulsory Secondary students (323 boys and 285 girls; $M_{\text {age }}=14.99$, $S D=1.58$ ), who completed a 12-session intervention in Physical Education class according to the guidelines proposed for this model-based practice. The confirmatory factor analysis showed a good fit for the 6-ítem and 1-factor structure, which was invariant across gender and age. The internal consistence analysis reflected adequate values. The three lineal regression analyses revealed that sport education predicted positively perceived competence, sport engagement and fair play, respectively. The
\end{abstract}


Physical Education Season Survey is shown to be a valid and reliable measure to assess the six core features of Sport Education in the Spanish Physical Education context.

Keywords: models-based practice, sport instruction models, sport pedagogy, psychometric examination, design and validation.

Correspondencia: Rafael Burgueño Menjibar, Facultad de Ciencias de la Educación. Universidad de Almería. Carretera de Sacramento s/n, 04120 La Cañada de San Urbano, (Almería), España. Tel: +34 950015 092. E-mail: $\underline{\text { rbm288@ual.es. }}$

La Educación Deportiva (ED; Siedentop, 1994) nace como respuesta a la falta de experiencias de aprendizaje auténticas y significativas proporcionadas por los enfoques centrados en la habilidad para la enseñanza del deporte en la asignatura de Educación Física (EF) (Hastie \& Wallhead, 2016). Este modelo basado en la práctica se define de acuerdo a seis características estructurales (i.e., temporadas, afiliación, competición regular, evento culminante, registro de datos y festividad) extraídas del deporte contemporáneo con la finalidad de ser aplicadas en el proceso de enseñanza-aprendizaje deportivo que tiene lugar en clase de EF (Siedentop, 1994). En este sentido, la presente investigación tiene como propósito analizar las propiedades psicométricas de una medida que valora la percepción del alumnado en relación con las características estructurales de la ED en el contexto español de la EF.

La ED es un modelo pedagógico que tiene como meta educar a estudiantes como deportistas competentes, cultos y comprometidos con su práctica deportiva (Siedentop, 1994). De acuerdo con Siedentop (1994), un estudiante competente tendrá la suficiente habilidad motriz para participar en los deportes de manera satisfactoria, teniendo la posibilidad de entender y ejecutar con éxito estrategias adecuadas a la hora de hacer frente a la complejidad del juego. Asimismo, un alumno culto entenderá y valorará las reglas de juego, a los árbitros y a las tradiciones deportivas, así como discernirá entre una buena y mala práctica deportiva dentro y fuera del campo. De igual forma, un discente entusiasmado sería aquel que participa de tal manera que preserva, protege y mejora la cultura deportiva, estando, a su vez, comprometido con el deporte.

Para tal finalidad, la ED se fundamenta en una enseñanza centrada en el estudiante y en una pedagogía constructivista y cooperativa (Siedentop, 1994). Es más, se caracteriza por un elevado apoyo a la autonomía (Medina-Casaubón \& Burgueño, 2017) y un clima orientado a la tarea (Hastie, Sinelnikov, Wallhead, \& Layne, 2014). Su metodología se materializa con el trabajo en grupos reducidos y juegos modificados, situados en la zona de desarrollo próximo del estudiante (Hastie \& Wallhead, 2016), con la finalidad de mejorar tanto el componente técnico-táctico y conceptual como la toma de decisiones, la responsabilidad y la autonomía del aprendiz (Siedentop, 1994).

Siedentop (1994) definió la ED en base a una serie de características estructurales extraídas del deporte institucionalizado actual, las cuales no se encuentran habitualmente en la enseñanza deportiva en la asignatura de EF. Así, Siedentop (1994) describió los siguientes seis rasgos innegociables: a) temporada, referida a una mayor duración de la unidad didáctica convencional para favorecer el aprendizaje de las habilidades deportivas; b) afiliación, referida a la agrupación del estudiante en un equipo de modo permanente durante toda la temporada; c) 
competición regular, referida a la combinación de períodos de entrenamiento, destinados a la práctica de habilidades deportivas, con períodos de competición, orientados a la aplicación de las habilidades deportivas aprendidas; d) evento culminante, referida a la realización de un acontecimiento final para decidir el ganador y entregar los premios para elogiar todos los logros obtenidos; e) registro de datos, referido a la anotación regular de información relacionada con el juego limpio, análisis de juego o desempeño del juego; f) festividad, referida a la naturaleza festiva del deporte que debe envolver el proceso de enseñanza-aprendizaje deportivo en EF a través de la simbología (colores, himnos, etc.), rituales (darse la mano, foto de alineación, etc.) y celebraciones, tal y como sucede en los eventos deportivos. No obstante, habría que tener en consideración que cada temporada de ED posee un carácter único dado que depende tanto de la interpretación como de la prestación del docente. En este sentido, el profesor puede implementar ciertos principios propuestos para este modelo pedagógico pero, a su vez, omitir otros, dando lugar a una "versión rebajada" de este modelo basado en la práctica (Curtner-Smith, Hastie, \& Kinchin, 2008). Ante esta realidad, resulta crucial que la fidelidad al modelo y sus características se encuentren bien establecidas cuando se desea investigar sobre un modelo pedagógico determinado (Hastie \& Casey, 2014).

La literatura previa se ha centrado en comprobar si la ED favorecía las tres metas que persigue este modelo como son el desarrollo de la competencia, la cultura deportiva y el entusiasmo del estudiante (Hastie, Martínez de Ojeda, \& Calderón, 2011; Hastie \& Wallhead, 2016). Concerniente a la percepción de competencia, se informó de un incremento sobre la competencia motriz percibida (Cuevas, García-López, \& Contreras, 2015; Mesquita, Rodrigues-Pereira, Araújo, Farias, \& Rolim, 2016), lo cual se mostró en consonancia tanto con la mejora en el nivel de habilidad técnico (Meroño, Calderón, \& Hastie, 2016; Pereira et al., 2015) como con un mayor rendimiento táctico y toma de decisiones (Mahedero, Calderón, Arias-Estero, Hastie, \& Guarino, 2015) constatado a nivel observacional. Respecto a la cultura deportiva, este modelo pedagógico mejoró el conocimiento deportivo (Calderón, Hastie, \& Martínez-de-Ojeda, 2010), el juego limpio (Wahl-Alexander, Sinelnikov, \& Curtner-Smith, 2017) y generó una conducta más ética del alumnado (Harvey, Kirk, \& O’Donovan, 2014). En relación con la participación entusiasta del alumnado, se observó un mayor nivel de entusiasmo del estudiante en clase (Mesquita et al., 2016). Es más, Méndez-Giménez, Martínez-de-Ojeda, y Valverde-Pérez (2016) descubrieron que el aumento en el entusiasmo fue ligeramente mayor cuando se usó material auto-construido por el alumnado en lugar de material convencional. Esta participación más entusiasta del alumnado, se reflejó en indicadores tales como una mayor motivación intrínseca (Burgueño, Medina-Casaubón, Morales-Ortiz, Cueto-Martin, \& SáchezGallardo, 2017) o un mayor compromiso deportivo en el aula (Calderón, Martínez-de-Ojeda, \& Hastie, 2013; Meroño, Calderón, \& Hastie, 2015).

Hasta este momento, la única medida encontrada en la literatura para valorar la percepción de las características estructurales de la ED ha sido la Physical Education Season Survey (Mohr, Townsend, Rairigh, \& Mohr, 2003). Esta medida consta de 12 ítems divididos en dos secciones. La primera sección consta de 6 ítems creados para medir los seis rasgos innegociables de la ED (i.e., temporada, afiliación, competición regular, evento final, registro de datos y festividad). La segunda sección consiste en seis cuestiones referidas a la percepción de competencia, al conocimiento de las reglas de juego y respeto por las reglas como indicadores de la cultura deportiva y, de la percepción del nivel de compromiso. A pesar de que el instrumento ha sido 
traducido y utilizado al contexto ruso con estudiantes educación primaria (Hastie \& Sinelnikov, 2006), al contexto anglosajón con estudiantes de educación primaria y secundaria (FittipaldiWert, Brock, Hastie, Arnold, \& Guarino, 2007) o al contexto español con estudiantes de primaria (Calderón et al., 2010; Gutiérrez, García-López, Chaparro-Jilete, \& Fernández-Sánchez, 2014), no se mostraron evidencias ni de validez ni de fiabilidad de la Physical Education Season Survey. No obstante, Kinchin, Wardle, Roderick, y Sprosen (2004), en el contexto británico con estudiantes de educación secundaria, ofrecieron evidencias de validez basada en el contenido del instrumento mediante una evaluación subjetiva del instrumento realizada por parte de un grupo de expertos, en cambio, no proporcionaron evidencias de fiabilidad de esta escala. En este sentido, hasta este momento, no se tiene constancia de investigaciones que hayan proporcionado evidencias de validez basadas en la estructura interna, evidencias de fiabilidad o evidencias de validez basada en la relación con otras variables que apoyen el adecuado funcionamiento psicomérico de la Physical Education Season Survey en la valoración de la percepción de los seis rasgos nucleares que definen a la ED.

Por consiguiente, esta investigación tuvo como objetivo analizar las propiedades psicométricas de la primera sección del Physical Education Season Survey con el propósito de medir de manera válida y fiable las seis características estructurales e innegociables de la ED en estudiantes de educación secundaria obligatoria y educación secundaria post-obligatoria.

\section{Método}

\section{Participantes}

Los participantes fueron 608 estudiantes de educación secundaria obligatoria y de educación secundaria post-obligatoria (323 chicos y 285 chicas) con una edad comprendida entre 12 y 19 años $\left(M_{\text {edad }}=14.99, D T=1.58\right)$ pertenecientes a centros educativos públicos de una ciudad al sureste español. A su vez, tomaron parte 26 profesores de EF en formación inicial (15 hombres y 11 mujeres) con una edad comprendida entre 20 y 23 años $\left(M_{\text {edad }}=21.74, D T=\right.$ 1.31) - matriculados en el grado de Ciencias de la Actividad Física y el Deporte de una universidad situada al sureste de España - quienes llevaron a la práctica el programa de intervención en cada uno de los centros educativos participantes.

\section{Medidas}

ED. Se utilizó la versión española (Calderón et al., 2010) de la Physical Education Season Survey (Mohr et al., 2003) para medir la percepción de la ED. El instrumento consta de un total de 6 ítems para medir la característica estructural de temporada, ítem 1, ("me he divertido a lo largo de la temporada"), afiliación social, ítem 2, ("me ha gustado pertenecer siempre al mismo equipo a lo largo de toda la temporada"), registro de datos, ítem 3, ("me ha gustado registrar los puntos y los resultados durante los partidos"), competición formal, ítem 4, ("me ha gustado que pudiéramos practicar para después competir"), evento culminante, ítem 5, ("me ha gustado el día de la competición final") y de festividad, ítem 6, ("me ha gustado celebrar el día de la festividad en el que se entregan los premios"). Cada ítem se responde usando una escala-tipo-Likert de 5 puntos, desde 1 (mínimo) a 5 (máximo). 
Competencia. Se utilizó la versión española adaptada a la EF (Moreno, González-Cutre, Chillón-Garzón, \& Parra-Rojas, 2008) de la Basic Psychological Needs in Exercise Scale (Vlachopoulos \& Michailidou, 2006) para medir la competencia (e.g. "realizo los ejercicios eficazmente) mediante 4 ítems. Cada ítem se responde usando una escala-tipo-Likert de 7 puntos, desde 1 (totalmente en desacuerdo) a 7 (totalmente de acuerdo).

Juego Limpio y Compromiso Deportivo. Se utilizó la versión española (Martín-Albo, Núñez, Navarro-Izquierdo, \& González-Ruiz, 2006) de la Multidimensional Sportspersonship Orientations Scale (Vallerand, Brière, Blanchard, \& Provencher, 1997) para medir tanto el juego limpio (e.g. "después de una victoria, reconozco el trabajo de mi adversario") como el compromiso deportivo (e.g. "no me rindo, ni siquiera después de cometer muchos errores) mediante 5 ítems. Cada ítem se responde usando una escala-tipo-Likert de 5 puntos, desde 1 (no) a 5 (sí).

\section{Procedimiento}

Este trabajo obtuvo la aprobación del Comité de Bioética en Investigación Humana de la Universidad de Granada, además de la autorización del equipo directivo de todos los centros educativos participantes y el consentimiento informado de los progenitores de cada estudiante que tomó parte en este estudio. El instrumento fue administrado a la conclusión del programa de intervención en el aula. El personal investigador informó del carácter voluntario y anónimo en la participación, además de enfatizar en la ausencia de respuestas correctas y falsas, dado que sólo se quería conocer su opinión. Además, se estuvo a disposición de los encuestados para solucionar cualquier tipo de duda emergida durante el proceso de cumplimentación del instrumento. El tiempo aproximado de compleción del cuestionario fue de 10 minutos.

\section{Programa de Intervención}

El personal investigador junto con el profesorado de EF diseñaron una intervención de acuerdo con las premisas propuestas por Siedentop, Hastie, y Van der Mars (2011) para la ED. Dicha intervención se desarrolló en horario habitual de EF durante seis semanas, 12 sesiones de 55 minutos, con dos sesiones semanales, para un total de 660 minutos de período instructivo. Acorde con las recomendaciones de Siedentop et al. (2011), la intervención tuvo tres fases principales, donde el alumnado fue gradualmente asumiendo más responsabilidades a medida que la temporada avanzaba: a) una fase de desarrollo de habilidades, incluyendo una sesión introductoria y dos clases dirigidas por el profesor; b) una fase de pre-temporada o de práctica autónoma, la cual intercaló períodos de entrenamiento de las habilidades técnico-tácticas deportivas con períodos competitivos $\mathrm{y}, \mathrm{c}$ ) una fase de competición formal, incluyendo una competición regular y un evento culminante. 
Tabla 1. Principales Contenidos y Actividades del Programa de Intervención por Sesiones

\begin{tabular}{|c|c|c|c|}
\hline Sesión & Contenido de la sesión & Rol docente & Rol discente \\
\hline \multicolumn{4}{|c|}{ Desarrollo de habilidades: Fase de práctica dirigida por el docente } \\
\hline 1 & $\begin{array}{l}\text { Presentación de ED. Creación de equipos. Asignación } \\
\text { de roles. Elección del color de vestimenta y escudo. }\end{array}$ & Líder & Participante activo \\
\hline 2 & $\begin{array}{l}\text { Familiarización con la ED. Entrenamiento mediante } \\
\text { juegos modificados. }\end{array}$ & Líder & Subordinado \\
\hline 3 & $\begin{array}{l}\text { Familiarización con la ED. Entrenamiento mediante } \\
\text { juegos modificados. }\end{array}$ & Líder & Subordinado \\
\hline \multicolumn{4}{|c|}{ Pre-temporada: Fase de practica autónoma del alumnado } \\
\hline 4 & $\begin{array}{l}\text { Entrenamiento mediante juegos modificados. Torneo } \\
\text { pre-temporada. }\end{array}$ & Guía/ Mediador & Resolución problemas \\
\hline 5 & $\begin{array}{l}\text { Entrenamiento mediante juegos modificados. Torneo } \\
\text { pre-temporada. }\end{array}$ & Guía/ Mediador & Resolución problemas \\
\hline 6 & $\begin{array}{l}\text { Entrenamiento mediante juegos modificados. Torneo } \\
\text { pre-temporada. }\end{array}$ & Guía/ Mediador & Resolución problemas \\
\hline 7 & $\begin{array}{l}\text { Entrenamiento mediante juegos modificados. Torneo } \\
\text { pre-temporada. }\end{array}$ & Guía/ Mediador & Resolución problemas \\
\hline 8 & $\begin{array}{l}\text { Entrenamiento mediante juegos modificados. Torneo } \\
\text { pre-temporada. }\end{array}$ & Guía/ Mediador & Resolución problemas \\
\hline \multicolumn{4}{|c|}{ Competición formal } \\
\hline 9 & Competición (fase regular) & Guía/ Mediador & Resolución problemas \\
\hline 10 & Competición (fase regular) & Guía/ Mediador & Resolución problemas \\
\hline 11 & Competición (fase regular) & Guía/ Mediador & Resolución problemas \\
\hline 12 & $\begin{array}{l}\text { Competición (final y, tercer y cuarto puesto). Evento } \\
\text { festivo. Entrega de diplomas. }\end{array}$ & Maestro de ceremonia & Participante activo \\
\hline
\end{tabular}

Nota: ED = Educación Deportiva.

\section{Análisis Estadístico}

Los datos fueron tratados estadísticamente con el Statistical Package for the Social Sciences (IBM SPSS Statistics for Window, version 22.0; Armonk, NY, USA) y su extensión para analizar modelos de ecuaciones estructurales AMOS (version 22.0; Armonk, NY, USA). Se calcularon los estadísticos descriptivos, así como la correlación bivariada de Pearson para cada uno de los seis ítems. La validez basada en la estructura interna fue examinada mediante un análisis factorial confirmatorio y un análisis multi-grupo de invariancia. Para efectuar el análisis factorial confirmatorio, se seleccionó el método de máxima verosimilitud con la técnica no paramétrica de bootstrapping (Hair, Black, Babin, \& Anderson, 2010), dada la ausencia de normalidad multivariante (coeficiente de Mardia $=18.28, p<.01$ ). Esta técnica no paramétrica 
permite solventar la violación del supuesto de normalidad multivariante, además de calcular el error típico de cada parámetro estadístico mediante la replicación aleatoria de 5000 muestras a partir de la muestra original (Hair et al., 2010). La bondad del ajuste fue evaluada por medio de diversos índices de ajuste: el ratio chi-cuadrado y grados de libertad $\left(\chi^{2} / g l\right)$, el Comparative Fit Index (CFI), el Tucker-Lewis Index (TLI), el Standardized Root Mean Square Residual (SRMR) y el Root Mean Square Error of Approximation (RMSEA) con su intervalo de confianza al 90\% (IC90\%). Hu y Bentler (1999) consideran como adecuados valores menores que 5 para el $\chi^{2} / g l$, mayores que .95 para CFI y TLI, menores que .080 para SRMR e inferiores a .050 para RMSEA. Teniendo en consideración que tales puntos de corte pueden resultar excesivamente restrictivos a la hora de juzgar la bondad del ajuste de cualquier modelo factorial, West, Taylor, y Wu (2012) sostienen que valores menores que .080 para RMSEA podrían ser indicativos de un ajuste razonable, siempre y cuando el límite superior de su intervalo de confianza al $90 \%$ sea inferior a .10. Los pesos de regresión estandarizados son apropiados con valores mayores que $.50 \mathrm{y}$ las covarianzas residuales estandarizadas con valores absolutos menores que 2.00 (Hair et al., 2010). La invariancia del instrumento tanto por género como por edad fue inspeccionada mediante dos análisis multi-grupo de invariancia factorial de acuerdo con la propuesta metodológica de Gregorich (2006). Tal enfoque analiza cuatro modelos progresivamente restringidos para testar la invariancia configural (sin restricciones), la invariancia métrica (restricción en pesos de medida de los ítems), la invariancia fuerte (restricción en pesos de medida e interceptos de los ítems) y la invariancia estricta (restricción en pesos de medida, interceptos y varianzas de error de los ítems). La ausencia de diferencias estadísticamente significativas en el test $\chi^{2}$ entre cada uno de los cuatros modelos progresivamente restringidos es indicativo del carácter invariante del instrumento de medida (Fan \& Sivo, 2009). Respecto al análisis multi-grupo por edad, se establecieron dos grupos de edad a partir de la mediana. El primero de los grupos estuvo compuesto por 369 estudiantes con una edad entre 12 y 15 años $\left(M_{\text {edad }}=13.99, D T=0.98\right)$; mientras que el segundo grupo estuvo formado por 235 estudiantes con una edad entre 16 y 19 años $\left(M_{\text {edad }}=16.56, D T=0.95\right)$. La consistencia interna fue analizada, por una parte, mediante la estimación de alfa de Cronbach y del coeficiente de fiabilidad compuesta de Raykov, aceptables con valores superiores a .70 (Viladrich, Angulo-Brunet, \& Doval, 2017) y, por otra parte, con el cálculo de la varianza media extraída, apropiada con valores mayores que .50 (Hair et al., 2010). La validez basada en la asociación con otras variables fue examinada mediante tres análisis de regresión lineal, en donde, la ED fue introducida como variable independiente, mientras que la competencia percibida, el juego limpio y el compromiso deportivo fueron consideradas, respectivamente, como variables dependientes.

\section{Resultados}

\section{Análisis Factorial Confirmatorio}

El análisis factorial confirmatorio reveló aceptables índices de ajuste: $\chi^{2}(9, N=608)=$ $38.04, p<.001 ; \chi^{2} / g l=4.23 ; \mathrm{CFI}=.98 ; \mathrm{TLI}=.97 ;$ SRMR $=.023 ; \mathrm{RMSEA}=.073(\mathrm{IC} 90 \%=$ $.050, .098)$. Como muestra la Tabla 2 , los pesos de regresión estandarizados oscilaron entre .70 y .79, alcanzando cada uno de ellos el nivel de significación estadística $(p<.001)$. Las covarianzas residuales estandarizadas estuvieron comprendidas entre -1.08 y 1.12 . 
Tabla 2. Estadísticos Descriptivos, Varianza, Pesos de Regresión Estandarizados, Correlaciones Múltiples Cuadradas y Covarianzas Residuales Estandarizadas obtenidas por el Análisis Factorial Confirmatorio y, Correlaciones Bivariadas de Pearson entre Ítems

\begin{tabular}{lccccccccccccc}
\hline & $M$ & $S D$ & Asimetría & Curtosis & Varianza & $\lambda$ & CMC & 1 & 2 & 3 & 4 & 5 & 6 \\
\hline 1. Ítem 1 & 4.02 & 1.04 & -0.80 & 0.23 & $.56(.05)$ & $.69(.03)$ & $.47(.04)$ & - & $.57^{* *}$ & $.54^{* *}$ & $.53^{* *}$ & $.53^{* *}$ & $.48^{* *}$ \\
2. Ítem 2 & 4.08 & 0.97 & -0.73 & 0.28 & $.41(.04)$ & $.75(.03)$ & $.57(.04)$ & 1.12 & - & $.62^{* *}$ & $.55^{* *}$ & $.57^{* *}$ & $.55^{* *}$ \\
3. Ítem 3 & 4.21 & 0.94 & -0.90 & 0.19 & $.34(.03)$ & $.78(.02)$ & $.62(.03)$ & -0.01 & 0.55 & - & $.65^{* *}$ & $.62^{* *}$ & $.52^{* *}$ \\
4. Ítem 4 & 4.26 & 0.87 & -0.88 & 0.35 & $.28(.03)$ & $.78(.02)$ & $.62(.04)$ & -0.37 & -0.91 & 0.46 & - & $.64^{* *}$ & $.58^{* *}$ \\
5. Ítem 5 & 4.18 & 0.91 & -0.86 & 0.36 & $.31(.03)$ & $.79(.02)$ & $.62(.04)$ & -0.25 & -0.53 & -0.15 & 0.25 & - & $.60^{* *}$ \\
6. Ítem 6 & 4.23 & 0.90 & -0.98 & 0.75 & $.39(.03)$ & $.72(.03)$ & $.52(.04)$ & -0.41 & 0.31 & -1.08 & 0.37 & 0.71 & - \\
\hline
\end{tabular}

Nota: $\lambda=$ Peso de regresión estandarizado; $\mathrm{CMC}=$ Correlación múltiple cuadrada. Los números entre paréntesis representan el error estándar estimado por el bootstrapping. Los números en la parte inferior de los guiones representan las covarianzas residuales estandarizadas.

\section{Análisis de Invariancia Factorial}

La Tabla 2 muestra la ausencia de diferencias estadísticamente significativas en el test $\chi^{2}$ entre cada uno de los sucesivos modelos restringidos tanto para el análisis multi-grupo por género como para el análisis multi-grupo por edad. Por tanto, la hipótesis nula de invariancia factorial respecto al género y a la edad fue asumida para dicho instrumento de medida.

Tabla 3. Análisis Multi-Grupo de Invariancia Factorial

\begin{tabular}{lcccccccccc}
\hline \multicolumn{10}{c}{ Invariancia por Género } \\
\hline & $\chi^{2}$ & $g l$ & $\chi^{2} / g l$ & CFI & TLI & SRMR & RMSEA (IC90\%) & Comparación & $\Delta \chi^{2}$ & $\Delta g l$ \\
\hline 1. Invariancia configural & 55.30 & 18 & 3.07 & .979 & .965 & .030 & $.058(.041, .076)$ & - & - & - \\
2. Invariancia métrica & 59.08 & 23 & 2.57 & .980 & .974 & .031 & $.051(.035, .067)$ & 2 versus 1 & 3.78 & 5 \\
3. Invariancia fuerte & 66.87 & 29 & 2.31 & .979 & .978 & .032 & $.046(.032, .061)$ & 3 versus 2 & 7.78 & 6 \\
4. Invariancia estricta & 79.30 & 35 & 2.27 & .975 & .979 & .035 & $.046(.032, .059)$ & 4 versus 3 & 12.44 & 6 \\
\hline & & & \multicolumn{1}{c}{ Invariancia por Edad } & & & \\
\hline & $\chi^{2}$ & $g l$ & $\chi^{2} / g l$ & CFI & TLI & SRMR & RMSEA (IC90\%) & Comparación & $\Delta \chi^{2}$ & $\Delta g l$ \\
\hline 1. Invariancia configural & 54.39 & 18 & 3.02 & .979 & .965 & .025 & $.058(.041, .076)$ & - & - & - \\
2. Invariancia métrica & 60.20 & 23 & 2.62 & .979 & .972 & .026 & $.052(.036, .068)$ & 2 versus 1 & 5.81 & 5 \\
3. Invariancia fuerte & 68.37 & 29 & 2.36 & .977 & .977 & .027 & $.047(.033 .0 .62)$ & 3 versus 2 & 8.16 & 6 \\
4. Invariancia estricta & 78.60 & 35 & 2.25 & .975 & .978 & .028 & $.045(.032, .059)$ & 4 versus 3 & 10.23 & 6 \\
\hline
\end{tabular}

Nota: $\chi^{2}=$ Estadístico chi-cuadrado; $g l=$ Grados de libertad obtenidos del test chi-cuadrado; CFI = Comparative Fit Index; TLI = Tucker-Lewis Index; SRMR = Standardized Root Mean Square Residual; RMSEA = Root Mean Square Error of Approximation; $\Delta \chi^{2}=$ Incremento del estadístico chi-cuadrado; $\Delta g l=$ Incremento de grados de libertad.

$* p<.05$

(C) Psy, Soc, \& Educ, 2018, Vol. 10(2) 


\section{Análisis de Consistencia Interna}

El análisis de consistencia interna arrojó un alfa de Cronbach de .88 , un coeficiente de fiabilidad compuesta de Raykov de .89 y una varianza media extraída de .57 para la ED.

\section{Análisis de Regresión Lineal}

La Tabla 3 refleja que la ED predijo de manera positiva y estadísticamente significativa la competencia $(\beta=.57, p<.001)$, el juego limpio $(\beta=.37, p<.001)$ y el compromiso deportivo $(\beta=.47, p<.001)$. La varianza total explicada para cada uno de los tres modelos fue del $32 \%, 14 \%$ y $22 \%$, respectivamente.

Tabla 4. Análisis de Regresión Lineal para Predecir la Competencia, el Juego Limpio y el Compromiso Deportivo a partir de la ED

\begin{tabular}{lccccccccccccccc}
\hline & \multicolumn{4}{c}{ Competencia } & \multicolumn{4}{c}{ Juego Limpio } & \multicolumn{4}{c}{ Compromiso Deportivo } \\
\cline { 2 - 14 } & $\mathrm{B}$ & $\mathrm{ET}$ & $\beta$ & $\mathrm{t}$ & $\mathrm{R}^{2}$ & $\mathrm{~B}$ & $\mathrm{ET}$ & $\beta$ & $\mathrm{t}$ & $\mathrm{R}^{2}$ & $\mathrm{~B}$ & $\mathrm{ET}$ & $\beta$ & $\mathrm{t}$ & $\mathrm{R}^{2}$ \\
\hline $\begin{array}{l}\text { (constante) } \\
\text { Educación }\end{array}$ & 2.02 & .21 & - & 9.78 & .32 & 2.32 & .17 & - & 13.34 & .14 & 2.49 & .13 & - & 18.90 & .22 \\
Deportiva & .84 & .05 & $.57^{* * *}$ & 17.10 & & .41 & .04 & $.37^{* * *}$ & 9.87 & & .40 & .03 & $.47^{* * *}$ & 12.96 & \\
\hline
\end{tabular}

Nota: $\mathrm{B}=$ Coeficiente de regresión no estandarizado; $\mathrm{ET}=$ Error Típico de $\mathrm{B} ; \beta=$ Coeficiente de regresión estandarizado; $\mathrm{t}=$ Estadístico $\mathrm{t} ; \mathrm{R}^{2}=$ Varianza total explicada.

$* * * p<.001$

\section{Discusión}

El objetivo de este estudio fue examinar las propiedades psicométricas de la primera sección del Physical Education Season Survey para evaluar de modo válido y fiable las seis características innegociables de la ED en estudiantes de educación secundaria obligatoria y educación secundaria post-obligatoria. En su conjunto, los resultados mostraron evidencias que respaldaron tanto la validez como la fiabilidad de dicho instrumento para evaluar las características estructurales que definen a este modelo pedagógico.

Los resultados obtenidos mostraron evidencias de validez basadas en la estructura interna del instrumento. En concreto, los resultados del análisis factorial confirmatorio sustentaron de manera psicométrica la estructura unidimensional de 6 ítems pertenecientes a la Physical Education Season Survey y propuestos para evaluar las características estructurales que identifican a la ED. Los índices de ajuste reflejaron un adecuado ajuste. Asimismo, cada uno de los seis pesos de regresión estandarizados alcanzó el nivel de significación estadística y valores superiores a .50, sugiriendo su idoneidad para evaluar el constructo psicológico para el que fueron creados. Adicionalmente, el análisis de modificación de índices descartó la correlación de pares de error entre ítems, indicando la ausencia de especificaciones erróneas en la estructura interna del instrumento. Por otro lado, los análisis multi-grupo de invariancia factorial por género y por edad reflejaron el carácter invariante de la primera sección de la Physical Education Season Survey en el contexto español para medir las seis características estructurales e innegociables de la ED. Estos hallazgos tienen una especial importancia dado 
que apoyan el uso en alumnado español de este instrumento a la hora de analizar las posibles diferencias existentes en la percepción de tales características nucleares de este modelo pedagógico en alumnado tanto de diferente edad (de 12 a 18 años) como de diferente género. En referencia a los resultados del análisis de consistencia interna, éstos revelaron apropiados valores para alfa de Cronbach, coeficiente de fiabilidad compuesta de Raykov y varianza media extraída, evidenciando, en su conjunto, una adecuada consistencia interna del instrumento.

Los resultados de los tres análisis de regresión lineal aportaron evidencias de validez basadas en la asociación con otras variables. De acuerdo con los resultados de este trabajo, la ED predijo de modo positivo y estadísticamente significativo la competencia, el juego limpio y el compromiso deportivo. Estos hallazgos se muestran en consonancia con la meta educativa enunciada por Siedentop et al. (2011) para la ED, así como con los resultados empíricos encontrados en la literatura concerniente a cada variable. En este sentido, la ED, diseñada originalmente para favorecer experiencias deportivas auténticas del alumnado, puede proporcionar un clima orientado a la tarea y un elevado apoyo a la autonomía (Hastie et al., 2014; Medina-Casaubón \& Burgueño, 2017), lo que podría conducir al estudiante a experimentar consecuencias de carácter positivo como un mayor compromiso (Meroño et al., 2015), juego limpio (Wahl-Alexander et al., 2017) y competencia percibida (Cuevas et al., 2015). En su conjunto, estos resultados ofrecen respaldo a la robustez de este modelo pedagógico a la hora de lograr las tres principales metas perseguidas por este modelo basado en la práctica para el alumnado como son el desarrollo de la competencia motriz (medida con la competencia percibida), la cultura deportiva (medida con el juego limpio) y el entusiasmo (medido con el compromiso deportivo).

Pese a los resultados obtenidos en la presente investigación, una serie de limitaciones deben ser tomadas en consideración. Los participantes de este trabajo correspondieron a una muestra de conveniencia y, por consiguiente, los resultados no pueden ser generalizados al conjunto de la población. En este aspecto, se necesitan nuevos trabajos que examinen las propiedades psicométricas de la primera sección de la Physical Education Season Survey en nuevas poblaciones con la finalidad de sustentar los resultados obtenidos en el presente estudio. En esta misma línea, futuros trabajos deberían examinar las propiedades psicométricas de este instrumento en diferentes contextos como el anglosajón, el ruso o el portugués donde la ED está siendo implantada en la asignatura de la EF, o niveles educativos como Educación Primaria con el propósito de comprobar si la estructura factorial de este instrumento se adapta a otras culturas y niveles educativos. Además, futuras investigaciones podrían contribuir al desarrollo de esta medida aportando evidencias de validez desde otros enfoques psicométricos. Finalmente, se debe destacar que el estudio de las propiedades psicométricas y la validación de instrumentos en psicología se entiende como un proceso continuado dada la complejidad de evaluar los procesos cognitivos humanos.

En su conclusión, la presente investigación proporciona evidencias que respaldan la validez y la fiabilidad de la primera sección de la Physical Education Season Survey en el contexto español de la EF. En este sentido, a partir de este momento se puede disponer de un instrumento válido y fiable para valorar las características estructurales de la ED propuestas por Siedentop (1994) en clase de EF. Hasta este momento, ningún estudio había examinado de manera psicométrica este instrumento de medición de las características nucleares de este modelo pedagógico. En este aspecto, la primera sección de la Physical Education Season 
Survey puede considerarse como una medida psicométricamente robusta para evaluar las características estructurales que definen a este modelo basado en la práctica.

\section{Referencias}

Burgueño, R., Medina-Casaubón, J., Morales-Ortiz, E., Cueto-Martin, B., \& Sáchez-Gallardo, I. (2017). Sport education versus traditional teaching: Influence on motivational regulation in high school students. Cuadernos de Psicologia Del Deporte, 17(2), 87-98.

Calderón, A., Hastie, P. A., \& Martínez-de-Ojeda, D. (2010). Learning to teach sport education: Initial experience in elementary education. Cultura_Ciencia_Deporte, 6(5), 169-181. http://doi.org/10.12800/ccd.v5i15.103

Calderón, A., Martínez-de-Ojeda, D., \& Hastie, P. A. (2013). Students and teachers' perception after practice with two pedagogical models in Physical Education. International Journal of Sport Science, 32(9), 137-153. http://doi.org/10.5232/ricyde2013.03204

Cuevas, R., García-López, L. M., \& Contreras, O. (2015). Influence of the sport education model in the basic psychological needs. Cuadernos de Psicología Del Deporte, 15(2), 155-162.

Curtner-Smith, M. D., Hastie, P. A., \& Kinchin, G. D. (2008). Influence of occupational socialization on beginning teachers' interpretation and delivery of sport education. Sport, Education and Society, 13(1), 97-117. http://doi.org/10.1080/13573320701780779

Fan, X., \& Sivo, S. A. (2009). Using $\Delta$ goodness-of-fit indexes in assessing mean structure invariance. Structural Equation Modeling, 16(1), 54-69. http://doi.org/10.1080/10705510802561311

Fittipaldi-Wert, J., Brock, S. J., Hastie, P. A., Arnold, J. B., \& Guarino, A. J. (2007). Effects of a sport education curriculum model on the experiences of students With visual impairments. Palaestra, 24(3), 6-10.

Gregorich, S. E. (2006). Do self-report instruments allow meaningful comparisons across diverse population groups? Testing measurement invariance using the confirmatory factor analysis framework. Medical Care, 44(11 Suppl 3), S78-S94. http://doi.org/10.1097/01.mlr.0000245454.12228.8f

Gutiérrez, D., García-López, L. M., Chaparro-Jilete, R., \& Fernández-Sánchez, A. J. (2014). Sport education model in second grade. Teachers and students' perceptions. Cuadernos de Psicología Del Deporte, 14(2), 131-143.

Hair, J. F. J., Black, W. C., Babin, B. J., \& Anderson, R. E. (2010). Multivariate data analysis (7th ed.). Englewood Cliffs, NJ: Pearson Prentice Hall.

Harvey, S., Kirk, D., \& O’Donovan, T. M. (2014). Sport education as a pedagogical application for ethical development in physical education and youth sport. Sport, Education and Society, 19(1), 41-62. http://doi.org/10.1080/13573322.2011.624594

Hastie, P. A., \& Casey, A. (2014). Fidelity in models-based practice research in sport pedagogy: A guide for future investigations. Journal of Teaching in Physical Education, 33(3), 422-431. http://doi.org/10.1123/jtpe.2013-0141

Hastie, P. A., Martínez de Ojeda, D., \& Calderón, A. (2011). A review of research on Sport Education: 2004 to the present. Physical Education and Sport Pedagogy, 16(2), 103132. http://doi.org/10.1080/17408989.2010.535202 
Hastie, P. A., \& Sinelnikov, O. A. (2006). Russian students' participation in and perceptions of a season of Sport Education. European Physical Education Review, 12(2), 131-150. http://doi.org/10.1177/1356336X06065166

Hastie, P. A., Sinelnikov, O., Wallhead, T., \& Layne, T. (2014). Perceived and actual motivational climate of a mastery-involving sport education season. European Physical Education Review, 20(2), 215-228. http://doi.org/10.1177/1356336X14524858

Hastie, P. A., \& Wallhead, T. (2016). Models-based practice in physical education: The case for sport education. Journal of Teaching in Physical Education, 35(4), 390-399. http://doi.org/10.1123/jtpe.2016-0092

Hu, L., \& Bentler, P. M. (1999). Cutoff criteria for fit indexes in covariance structure analysis: Conventional criteria versus new alternatives. Structural Equation Modeling, 6(1), 1-55. http://doi.org/10.1080/10705519909540118

Kinchin, G. D., Wardle, C., Roderick, S., \& Sprosen, A. (2004). A survey of year 9 boys' perceptions of Sport Education in one English secondary school. Bulletin of Physical Education, 40(1), 27-40.

Mahedero, P., Calderón, A., Arias-Estero, J. L., Hastie, P. A., \& Guarino, A. J. (2015). Effects of student skill level on knowledge, decision making, skill execution and game performance in a mini-volleyball Sport Education season. Journal of Teaching in Physical Education, 34, 626-641. http://doi.org/10.1123/jtpe.2014-0061

Martín-Albo, J., Núñez, J. L., Navarro-Izquierdo, J. G., \& González-Ruiz, V. M. (2006). Validity of the Spanish version of the multidimensional sportpersonship orientations scale. Revista de Psicología Del Deporte, 15(1), 9-22.

Medina-Casaubón, J., \& Burgueño, R. (2017). Influence of a Sport Education season on motivational strategies in High School students: A Self-Determination Theory-based perspectiva. E-Balonmano.com: Journal of Sport Science, 13(2), 153-166.

Méndez-Giménez, A., Martínez-de-Ojeda, D., \& Valverde-Pérez, J. J. (2016). Valoración del alumnado y profesorado del material convencional y auto-construido: estudio longitudinal de diseño cruzado en Educación Deportiva. Retos. Nuevas Tendencias En Educación Física Y Recreación, 30, 20-25.

Meroño, L., Calderón, A., \& Hastie, P. A. (2015). Effect of a Sport Education season on psycological variables in youth swimmers. Cuadernos de Psicología Del Deporte, 15(2), $35-46$.

Meroño, L., Calderón, A., \& Hastie, P. A. (2016). Effect of Sport Education on the technical learning and motivational climate of junior high performance swimmers. RICYDE: Revista Internacional de Ciencias Del Deporte, 44(42), 182-198. http://doi.org/10.5232/ricyde2016.04407

Mesquita, I., Rodrigues-Pereira, J. A., Araújo, R., Farias, C., \& Rolim, R. (2016). Representação dos alunos e professora acerca do valor educativo do modelo de educação desportiva numa unidade didática de atletismo. Motricidade, 12(1), 26-42. http://doi.org/10.6063/motricidade.4213

Mohr, D. J., Townsend, S. J., Rairigh, R., \& Mohr, C. (2003). Students' perceptions of Sport Education when taught using pedagogical approach to Sport Education (PASE) planning and instructional framework. Research Quarterly for Exercise and Sport, 74(supplement), A51-A52. 
Moreno, J. A., González-Cutre, D., Chillón-Garzón, M., \& Parra-Rojas, N. (2008). Adaptación a la educación física de la escala de las necesidades psicológicas básicas en el ejercicio. Revista Mexicana de Psicología, 25(2), 295-303.

Pereira, J., Hastie, P. A., Araújo, R., Farias, C., Rolim, R., \& Mesquita, I. (2015). A comparative study of students' track and field technical performance in sport education and in a direct instruction approach. Journal of Sports Science and Medicine, 14(1), 118-127.

Siedentop, D. (1994). Sport Education: Quality PE through positive sport experiences. Champaign, IL: Human Kinetics.

Siedentop, D., Hastie, P. A., \& van der Mars, H. (2011). Complete guide to sport education (2nd ed.). Champaign, IL: Human Kinetics.

Vallerand, R. J., Brière, N. M., Blanchard, C., \& Provencher, P. (1997). Development and validation of the multidimensional sportspersonship orientations scale. Journal of Sport and Exercise Psychology, 19(2), 197-206. http://doi.org/10.1123/jsep.19.2.197

Viladrich, C., Angulo-Brunet, A., \& Doval, E. (2017). A journey around alpha and omega to estimate internal consistency reliability. Annals of Psychology, 33(3), 755-782. http://doi.org/10.6018/analesps.33.3.268401

Vlachopoulos, S. P., \& Michailidou, S. (2006). Development and initial validation of a measure of autonomy, competence, and relatedness in exercise: The basic psychological needs in exercise scale. Measurement in Physical Education and Exercise Science, 10(3), 179201. http://doi.org/10.1207/s15327841mpee1003_4

Wahl-Alexander, Z., Sinelnikov, O., \& Curtner-Smith, M. (2017). A longitudinal analysis of students' autobiographical memories of participation in multiple sport education seasons. European Physical Education Review, 23(1), 25-40. http://doi.org/10.1177/1356336X15624246

West, S. G., Taylor, A. B., \& Wu, W. (2012). Model fit and model selection in structural equation modeling. In R. H. Hoyle (Ed.), Handbook of Structural Equation Modeling (pp. 209-231). New York, NY: Guilford. 